\title{
Study of Healthcare Personnel with Influenza and other Respiratory Viruses in Israel (SHIRI): study protocol
}

Avital Hirsch ${ }^{1 *}$, Mark A. Katz ${ }^{1,2,3}$, Alon Laufer Peretz ${ }^{4}$, David Greenberg ${ }^{5}$, Rachael Wendlandt ${ }^{6}$, Yonat Shemer Avni ${ }^{7}$, Gabriella Newes-Adeyii, ${ }^{6}$ lan Gofer ${ }^{1}$, Maya Leventer-Roberts', Nadav Davidovitch ${ }^{8}$, Anat Rosenthal ${ }^{8}$, Rachel Gur-Arie ${ }^{8}$, Tomer Hertz ${ }^{9,10}$, Aharona Glatman-Freedman ${ }^{11,14}$, Arnold S. Monto ${ }^{3}$, Eduardo Azziz-Baumgartner ${ }^{12}$, Jill Morris Ferdinands ${ }^{12}$, Emily Toth Martin ${ }^{3}$, Ryan E. Malosh ${ }^{3}$, Joan Manuel Neyra Quijandría ${ }^{13}$, Min Levine ${ }^{12}$, William Campbell ${ }^{6}$, Ran Balicer ${ }^{1}$, Mark G. Thompson ${ }^{12}$ and on behalf of the SHIRI workgroup

\begin{abstract}
Background: The Study of Healthcare Personnel with Influenza and other Respiratory Viruses in Israel (SHIRI) prospectively follows a cohort of healthcare personnel (HCP) in two hospitals in Israel. SHIRI will describe the frequency of influenza virus infections among HCP, identify predictors of vaccine acceptance, examine how repeated influenza vaccination may modify immunogenicity, and evaluate influenza vaccine effectiveness in preventing influenza illness and missed work.
\end{abstract}

Methods: Cohort enrollment began in October, 2016; a second year of the study and a second wave of cohort enrollment began in June 2017. The study will run for at least 3 years and will follow approximately 2000 HCP (who are both employees and members of Clalit Health Services [CHS]) with routine direct patient contact. Eligible HCP are recruited using a stratified sampling strategy. After informed consent, participants complete a brief enrollment survey with questions about occupational responsibilities and knowledge, attitudes, and practices about influenza vaccines. Blood samples are collected at enrollment and at the end of influenza season; HCP who choose to be vaccinated contribute additional blood one month after vaccination. During the influenza season, participants receive twice-weekly short message service (SMS) messages asking them if they have acute respiratory illness or febrile illness (ARFI) symptoms. III participants receive follow-up SMS messages to confirm illness symptoms and duration and are asked to self-collect a nasal swab. Information on socio-economic characteristics, current and past medical conditions, medical care utilization and vaccination history is extracted from the CHS database. Information about missed work due to illness is obtained by self-report and from employee records. Respiratory specimens from self-collected nasal swabs are tested for influenza A and B viruses, respiratory syncytial virus, human metapneumovirus, and coronaviruses using validated multiplex quantitative real-time reverse transcription polymerase chain reaction assays. The hemagglutination inhibition assay will be used to detect the presence of neutralizing influenza antibodies in serum.

Discussion: SHIRI will expand our knowledge of the burden of respiratory viral infections among HCP and the effectiveness of current and repeated annual influenza vaccination in preventing influenza illness, medical utilization, and missed workdays among HCP who are in direct contact with patients.

Trial registration: NCT03331991. Registered on November 6, 2017.

\footnotetext{
* Correspondence: avitalhi@clalit.org.il

${ }^{1}$ Chief Physician's Office, Clalit Health Services, Clalit Research Institute, Tel

Aviv, Israel

Full list of author information is available at the end of the article
}

(c) The Author(s). 2018 Open Access This article is distributed under the terms of the Creative Commons Attribution 4.0 International License (http://creativecommons.org/licenses/by/4.0/), which permits unrestricted use, distribution, and reproduction in any medium, provided you give appropriate credit to the original author(s) and the source, provide a link to the Creative Commons license, and indicate if changes were made. The Creative Commons Public Domain Dedication waiver (http://creativecommons.org/publicdomain/zero/1.0/) applies to the data made available in this article, unless otherwise stated. 


\section{Background}

Healthcare personnel (HCP) are believed to be at increased risk of respiratory viral infections, with one in five estimated to be infected with seasonal influenza each year [1]. These estimates vary widely, however, depending on the extent of active surveillance and the use of serologic vs. molecular diagnostics. Respiratory infections are of particular concern among HCP because of the close contact of HCP with patients [2], and the risk of HCP transmitting respiratory viruses to others [3]. Although recent research suggests that certain subgroups of HCP, such as those who perform aerosol-generating procedures, may be at heightened risk of infection with influenza and other respiratory pathogens [4], incidence of infections among HCP is not well characterized across different occupations and responsibilities. Furthermore, although HCP often work while ill [5-7], the extent to which infected HCP transmit respiratory pathogens to patients is not clear [8]. Further research is needed on the frequency and types of interactions HCP have with patients when they have symptomatic, atypical, or asymptomatic $[1,9]$ influenza virus infections.

Vaccination of HCP against influenza is an important component of infection control in healthcare settings [10], but persistently low rates of vaccine uptake among HCP in most countries remains an international concern [10-12]. Numerous studies on the knowledge, attitudes, and practices (KAP) associated with influenza vaccine acceptance and rejection have been conducted among HCP in high-income countries in North America and Europe [13-16]. However, much less is known about the barriers to vaccine acceptance among HCP in countries in the Middle East [12]. In Israel, a high-income country, less than half (49\%) of HCP received influenza vaccine for the 2015-2016 influenza season; vaccine coverage was somewhat higher among hospital-based physicians (53\%) than nurses (46\%) [17]. More information is needed on the occupational, socio-demographic, and KAP factors that may explain variations in influenza vaccine acceptance in Israel, including how the personal KAP of HCP may impact on their promotion of influenza vaccination among their patients.

Although recent reviews confirm that the seasonal influenza vaccine is moderately effective in reducing the risk of influenza illness among adults [18], multiple gaps in knowledge remain about the preventive value of the vaccine among HCP [1]. To date, there has been only one randomized controlled trial of influenza vaccine efficacy among $\mathrm{HCP}$, and this study measured serologic outcomes only [19]. Given that vaccinated individuals are less likely to seroconvert after an influenza virus infection and so can appear as not having been infected [20,21], the use of serologic outcomes likely biases (and specifically, inflates) vaccine efficacy estimates.
Gaps are especially evident in our understanding of the value of influenza vaccine for reducing secondary adverse outcomes, including nosocomial infections among patients [22, 23] and missed work due to illness [24, 25]. Evidence is also needed to validate the potential of the vaccine in reducing the severity and duration of disease and diminish infectiousness among those who have breakthrough infections despite being vaccinated [24, 26]. Because HCP often receive influenza vaccine during multiple years, studies of HCP also provide an opportunity to examine how prior vaccinations may modify immunogenicity and vaccine effectiveness, and may provide answers to questions regarding the extent of residual protection and/ or negative vaccine interference across seasons [27-29].

Here we provide an overview of the design and methods of a prospective study of influenza vaccine effectiveness in HCP named "Study of Healthcare Personnel with Influenza and other Respiratory Viruses in Israel" (SHIRI). The SHIRI cohort will follow approximately $600 \mathrm{HCP}$, all of whom have direct patient contact, during at least three influenza seasons, and an additional $1400 \mathrm{HCP}$ during at least two influenza seasons.

Our study has four primary objectives: (1) to describe the frequency of respiratory, atypical (e.g., febrile only), and asymptomatic influenza virus infection among HCP; (2) to identify predictors of vaccine acceptance (and hesitancy) among HCP; (3) to examine how repeated influenza vaccination may modify immunogenicity; and (4) to evaluate influenza vaccine effectiveness in preventing influenza illness, associated missed work, and working while ill. Table 1 lists the knowledge gaps we identified within each of these aims and the study features intended to address these gaps.

The findings of this study will contribute to our knowledge about the burden of influenza and the vaccine effectiveness among HCP, and thus may influence a vaccination policy change in Israel and internationally.

\section{Methods/design Study design}

This prospective cohort study of influenza vaccine effectiveness in HCP is funded by the United States Centers for Disease Control and Prevention (US CDC). A steering committee, consisting of principal investigators from the two hospitals, Clalit Health Services (CHS), the Israeli Center for Disease Control, Abt Associates, and the US CDC was established and charged with making critical decisions related to the study. Additional investigators from the University of Michigan School of Public Health and the Ben Gurion University School of Public Health will advise on laboratory methods and qualitative methods related to the study. Study activities are described in Fig. 1. 
Table 1 Study Goals and Features Intended to Address Specific Knowledge Gaps

Knowledge Gap Study Feature

atypical illness, or asymptomatic infection

Studies of influenza illness among HCP using laboratory-confirmed outcomes are scarce.

Typical surveillance strategies have focused on acute respiratory illness using highly specific case definitions which overlook nonrespiratory and non-febrile manifestations of influenza disease.

Few studies have used both molecular and serologic diagnostics to assess the total burden of influenza virus infection among HCP.

It is unclear how differences in sex, age, occupation, and underlying health may contribute to the frequency of influenza illness among $\mathrm{HCP}$.

Further research is needed on whether specific HCP roles and responsibilities increase the risk of infection with influenza and

More information is needed on the impact of influenza illness on HCP's absence from work due to illness and working while ill.

2. Identification of predictors of vaccine acceptance (and hesitancy)

Most studies of HCP have focused on influenza vaccine uptake in specific seasons and less on behavior over multiple years.

Most studies of KAP associated

with influenza vaccination among HCP have been conducted in the United States or Western European countries. other respiratory pathogens.

Identification of symptomatic influenza virus infections with mqRT-PCR assay.

Usage of a broad case definition: "illness with cough, runny nose, body aches, or feverishness in the past seven days."

In addition to mqRT-PCR, 4-fold increases in HI from pre- to post-season will also be used to identify possible influenza virus infection among unvaccinated $\mathrm{HCP}$.

Usage of random stratified sampling to enroll a mixture of HCP by sex, age, and occupation. Assess underlying health status by self-report and medical record extraction.

Comparison of the frequency of ARFI (and infection with influenza and other respiratory viruses) by number of hours of direct patient care and by performance of aerosol-generating procedures (such as suction of fluids and tracheal intubation).

Assessment of the duration of illness, missed, and rescheduled work due to illness, hours worked during illness, and ability to do usual activities.

Description of how the frequency of influenza vaccination during the five years prior to enrollment and during the two to three years of participation in the cohort varies by sex, age, occupation, and socio-economic status.

This study is conducted in Israel, and will examine KAP topics including association between frequency of vaccination and perceived susceptibility to influenza, perceived benefits and risks of influenza vaccination, readiness to be vaccinated, and anticipated worry and regret about influenza vaccination decisions.

3. Examination of how repeated influenza vaccination may modify immunogenicity

Few studies have assessed the effects of repeated influenza vaccination across multiple seasons on immunogenicity.

Further research is needed on the mechanisms through which prior vaccination affects immunogenicity.

Further research is needed on whether repeated prior vaccination impacts cell mediated immune response to influenza vaccines.

Examination of how $\mathrm{HI}$ differs depending on the receipt of influenza vaccines up to ten years prior to the study for consistent health plans members.

Examination of whether any link between repeated vaccination and $\mathrm{HI}$ can be explained by HCP's "antibody landscape".

In a subset of participants who provide peripheral blood mononuclear cells before and after vaccination, examination of whether repeated prior vaccination is associated with suppression of B-cell and T-cell immunogenicity.

4. Evaluation of influenza vaccine effectiveness in preventing influenza illness and associated missed work and working while ill

Prior study of IVE among HCP used serologic outcomes, which are likely biased among vaccinees.

It is unclear whether influenza vaccines may reduce missed work due to influenza illness or reduce time spent working while ill (i.e., presenteeism) with influenza.

More information is needed on the extent to which prior vaccination may offer residual protection and/or interfere with IVE in subsequent seasons.

Further research is needed on whether the influenza vaccination may modify influenza disease severity and duration among those who become infected despite vaccination.

Estimate the effectiveness of the influenza vaccine in preventing mqRT-PCR confirmed influenza illness among HCP

Examine the hours of missed work and presenteeism between the dates of onset and resolution of influenza illness; apply these observations to estimate potential IVE in averting missed work or presenteeism.

Examination of IVE associated with combinations of current season vaccination and frequent vs. infrequent prior vaccinations.

Among HCP with influenza illness, examination of whether symptom severity and illness duration are lower among vaccinated vs. unvaccinated HCP.

Abbreviations: $H C P$ healthcare personnel, $m q R T-P C R$ multiplex quantitative real-time reverse transcription polymerase chain reaction, $H I$ hemagglutination inhibition, ARFI acute respiratory illness or febrile illness, KAP knowledge, attitudes, and practices, IVE influenza vaccine effectiveness

\section{Setting}

The study is being conducted among HCP at two hospitals in Israel: (1) Soroka University Medical Center (located in
Beer Sheva, a city in southern Israel), and (2) Beilinson Hospital (located in Petah Tivka, a city in central Israel) (Table 2). Both hospitals are managed and primarily staffed 


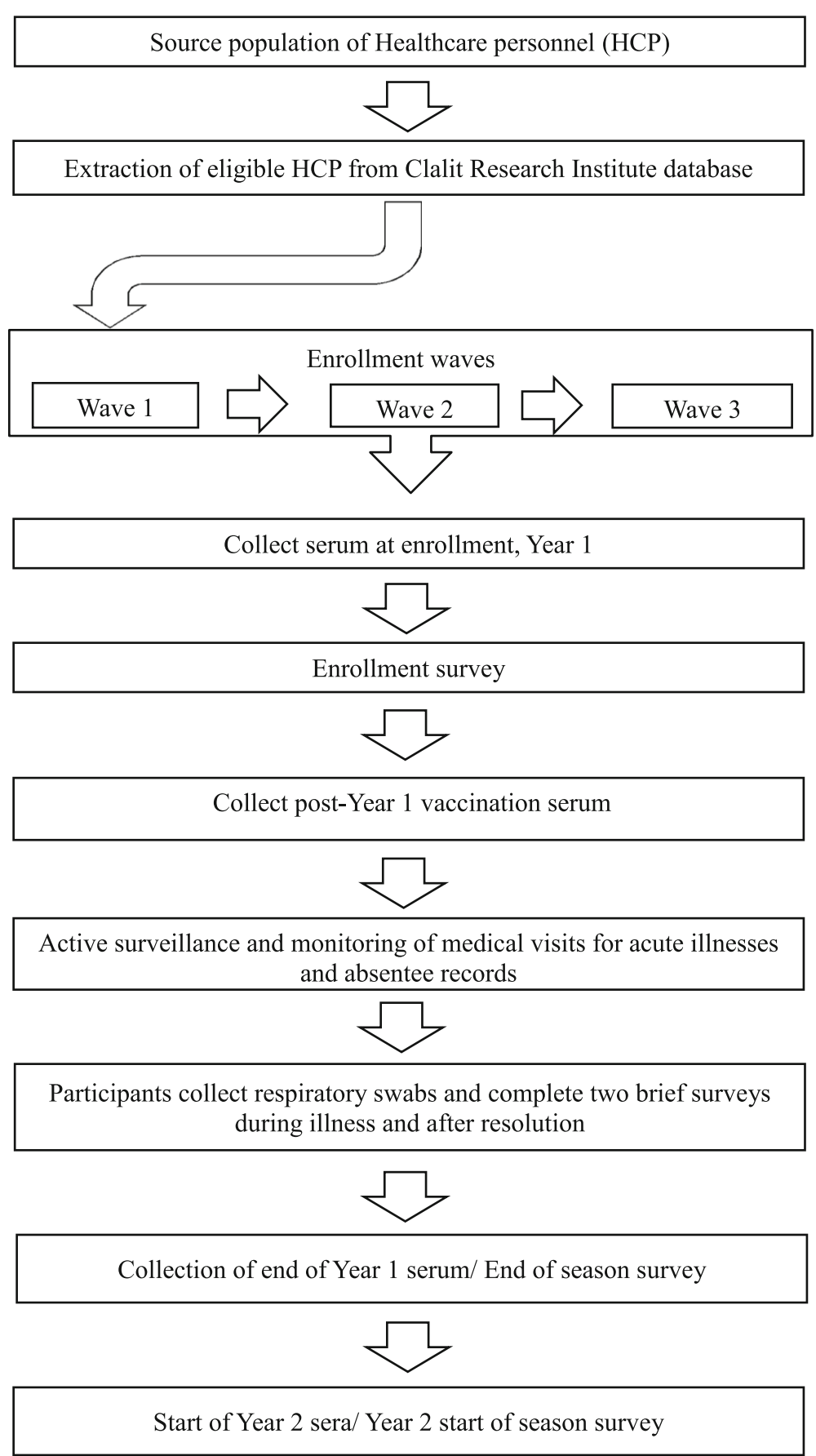

Fig. 1 Steps in Recruitment, Enrollment, and Follow-up

by CHS, the largest insurer and integrated care provider in Israel with over 4.4 million members in 2017, which constitute over $50 \%$ of the population.

\section{Participants}

\section{Eligibility criteria}

Eligible participants include HCP employed at a participating hospital who meet the following criteria: at least
18 years old; work full-time ( $\geq 30 \mathrm{~h}$ per week); and have routine direct hands-on or face-to-face contact with patients (within one meter) as part of a typical work shift. These eligibility criteria are similar to previous studies of HCP with direct patient contact [24,30]. Participation is offered to physicians, nurses, respiratory therapists, physical therapists, unit clerks, radiograph technicians, medical assistants, transporters, and other 
Table 2 Study Sites

\begin{tabular}{|c|c|c|c|c|c|}
\hline Name of study site & Location & Number of employees & Number Of beds & $\begin{array}{l}\text { Number of eligible } \\
\text { participants, } 2016^{c}\end{array}$ & $\begin{array}{l}\text { Number of eligible } \\
\text { participants, } 2017^{\text {d }}\end{array}$ \\
\hline Soroka University Medical Center & Beer Sheva, Israel & $4300^{b}$ & $1074^{\mathrm{a}}$ & 2436 & 2853 \\
\hline Beilinson Hospital & Petah Tikva, Israel & $5500^{b}$ & $850^{\mathrm{b}}$ & 1988 & 2260 \\
\hline
\end{tabular}

${ }^{a}$ Data as of 2016; Data were extracted from Israeli Ministry of Health reports

${ }^{b}$ Data of 2017; Data were extracted from employee records from the CHS EHR and Israeli Ministry of Health reports

${ }^{c}$ Recruitment in 2016 began in October, when nationwide vaccination was already in progress. HCP who had already been vaccinated in the current season were not eligible for the study

${ }^{d}$ Excludes participants enrolled in 2016

Number of beds data was obtained from Israeli Ministry of Health reports

Abbreviations: CHS Clalit Health Services, EMR electronic medical record, HCP healthcare personnel

HCP who have direct contact with patients. All participants must also be members of CHS and have had continuous membership in CHS for at least 1 year prior to enrollment (in order to access medical and vaccination records). HCP are invited to join the study whether or not they intend to be vaccinated. $\mathrm{HCP}$ are excluded if they received the current season's flu vaccine more than $48 \mathrm{~h}$ before enrollment. If enrollment goals are not met, some enrollment restrictions can be eliminated (e.g., requiring prior year membership in the Clalit health plan, working, $\geq 30 \mathrm{~h}$ per week, or plans to stay at the facility for at least 2 years) and differences in outcomes by these characteristics can be examined analytically.

Eligibility is determined by reviewing CHS's electronic medical record (EMR), which contains information on all of the eligibility criteria listed above except for the number of working hours, which is determined in the recruitment phone call. EMR data is extracted at the Clalit Research Institute, the research arm of CHS. Screening information is logged in the Research Electronic Data Capture (REDCap), a common database (described below).

\section{Recruitment}

In order to recruit HCP with diverse socio-demographic characteristics, occupational responsibilities, and influenza vaccination histories, eligible $\mathrm{HCP}$ were invited to participate using a stratified sampling strategy that targeted eligible participants in groups (strata) categorized by sex, age, occupation, and previous year influenza vaccination status. This systematic approach was intended to minimize convenience sampling, which can introduce both known and unknown biases. Each group includes individuals with unique characteristics known to potentially affect influenza vaccine immunogenicity and effectiveness.

At CHS, a list of eligible HCP at each facility was generated and categorized into groups based on age (18-34, $35-49$, $\geq 50$ years old), sex, occupational categories (1Physicians; 2 - Nurses, medical therapists, and professional technicians; and 3 - Medical assistants and support staff), and prior season influenza vaccination status (yes vs. no). Thus, prior to each year of the study, the sample is drawn from 36 unique strata $(2$ sexes * 3 age groups * 3 occupation groups *2 prior season vaccination status groups). This stratified sampling strategy will be applied to potential new participants each year.

At each hospital, goals are set for the minimum and maximum number of enrollees per strata; then, recruitment is implemented in three waves. During the first wave of recruitment, HCP from the 36 strata are invited at random to participate in the study. Next, in Wave 2, we identify study strata where additional recruitment is needed and expand direct invitations to additional $\mathrm{HCP}$ who have not yet been invited to join the study. In strata where all HCP have been contacted but recruitment goals have not been met, we will recruit similar HCP, prioritizing $\mathrm{HCP}$ who are similar in previous vaccination status, followed by age, sex, and lastly by profession. Finally, in Wave 3, we accept volunteers to meet the total sample goals for each facility. All potential enrollees will be recorded in a recruitment log in order to track invitations, acceptance, and refusal and reasons for refusal.

\section{Timeline of enrollment}

A total cohort of $2000 \mathrm{HCP}$ will be enrolled over a three-year period. Enrollment periods are targeted prior to influenza seasons. During Year 1 of the study, which targeted the 2016/2017 influenza season, enrollment could not begin until October 6, 2016 due to funding and institutional approval delays. Recruitment continued until the start of the influenza season (December 4, 2016) for a total of 8 weeks. This recruitment period included a month of frequent Israeli national holidays (October 2016), during which enrollment was challenging because many HCP were on vacation. In Year 1 of the study, 596 HCP were enrolled, and will contribute to Years 1, 2 and 3 of this 3 -year cohort study. Enrollment in Year 2 began in June 2017. New enrollees in Year 2 will contribute to Years 2 and 3 of the study.

\section{Active surveillance}

During the influenza season, participants receive twiceweekly short message service (SMS) messages asking them to confirm whether they have acute illness symptoms, 
defined as one or more of the following symptoms within the past 7 days: cough, runny nose, body aches, or feverishness. In addition to these SMS messages, participants are asked to contact the study staff immediately when they experience symptoms of acute illness. If a participant reports an acute illness, he or she is asked follow-up questions about specific symptoms and date of onset. Participants are first contacted by SMS message. If they do not respond, they are contacted by telephone. If participants do not respond to phone calls, an attempt is made to contact them in-person. Ill participants receive follow-up SMS messages about whether the illness has resolved. Once an illness resolution is reported by a participant, he or she is sent five follow-up questions by SMS message about illness presentation, duration, and impact on work attendance. These surveillance activities are described in Fig. 2.

The start and end of active surveillance is determined by the study investigators and steering committee based on historical patterns for seasonal influenza circulation and available clinical and surveillance indicators of laboratoryconfirmed influenza virus circulation in Israel. In the first year of the study, active surveillance started on December 4, 2016 and continued through March 23, 2017.

\section{Data collection}

We collect data for all participants from multiple sources. Key variables and their sources in the years prior to the study and during each study year are summarized in Table 3.

\section{Electronic medical records}

Information on socio-demographic characteristics, current medical conditions, medical history, medical care utilization, and influenza vaccination history are extracted from the CHS EMR at enrollment and at the end of each study year. For all participants, chronic medical conditions are identified using a combination of the International Classification of Diseases, Ninth Revision (ICD-9) codes from inpatient and outpatient records, International Classification of Primary Care Coding (ICPC), physician-entered free text diagnoses from outpatient medical records, and electronic chronic disease registries maintained by the Clalit Research Institute (details in Additional file 1: Annex 1 and Annex 2).

Routine influenza vaccine administration for CHS HCP members who receive the vaccine is recorded in the health fund's EMR. Because the vaccine is offered free of charge for members in CHS hospitals and clinics, nearly all of those who are vaccinated do so in such settings. Participants are considered vaccinated for a specific season if they received influenza vaccine from September 1 through March 31 of the relevant influenza season. To date, influenza vaccine has not been offered in Israel prior to September 1 . We document prior vaccine history, according to EMR records, from the the 2006-2007 season, 10 years prior to the first year of our study.
In addition, we document medical visits for ARFI during weeks of active surveillance in every study year. We also document medically attended ARFI in the 10 years prior to enrollment, distinguishing between medical visits that occurred during the influenza season and those that occurred outside of the influenza season. Medically attended ARFI episodes during active surveillance and for years prior to enrollment are captured through evaluation of outpatient visits, emergency room visits, and hospital admissions. Criteria used for medically attended ARFI are described in Additional file 1: Annex 3.

Outpatient visits, emergency room visits, medications dispensed at CHS pharmacies, and hospital admissions are regularly updated in the CHS EMR, making it possible to document medical visits nearly in real-time by recording ICD-9 codes and free text entered by physicians. We also access the EMR to document influenza antiviral medications and antibiotics dispensed during acute illnesses.

\section{Self-reported data}

At enrollment and at the beginning of each subsequent season, all participants complete a brief survey with questions about socio-demographic characteristics that are not available in the EMR, occupational responsibilities, health status, and KAP regarding seasonal influenza vaccination.

At the end of each influenza season, participants are asked to complete another brief survey that includes questions about their overall health and to describe previously unreported illnesses ('End-of-season survey'). Surveys are designed to be self-administered electronically through the Internet using the REDCap system.

\section{Work absenteeism}

During active surveillance, we document days of missed work associated with respiratory illnesses. Missed workdays are identified by self-report (as part of acute illness and illness follow-up SMS messages) and by periodic reviews of human resource department employee absentee records. Illness days are identified as all days between self-reported illness onset and illness resolution dates (from SMS surveillance messages or by direct phone screening). Thus, missed work due to illness includes days that participants directly reported missing due to illness and days of absence (according to employee records) between the illness onset and resolution dates.

\section{Laboratory methods \\ Nasal specimens}

When participating HCP report being ill with a respiratory illness during the influenza season, they are instructed to self-collect a nasal swab using a self-swabbing kit [31] that includes illustrated instructions, a nasal mid-turbinate 
[Sent weekly during flu season]

In the past 7 days, have you felt sick with a cough, runny nose, body aches or feverishness?

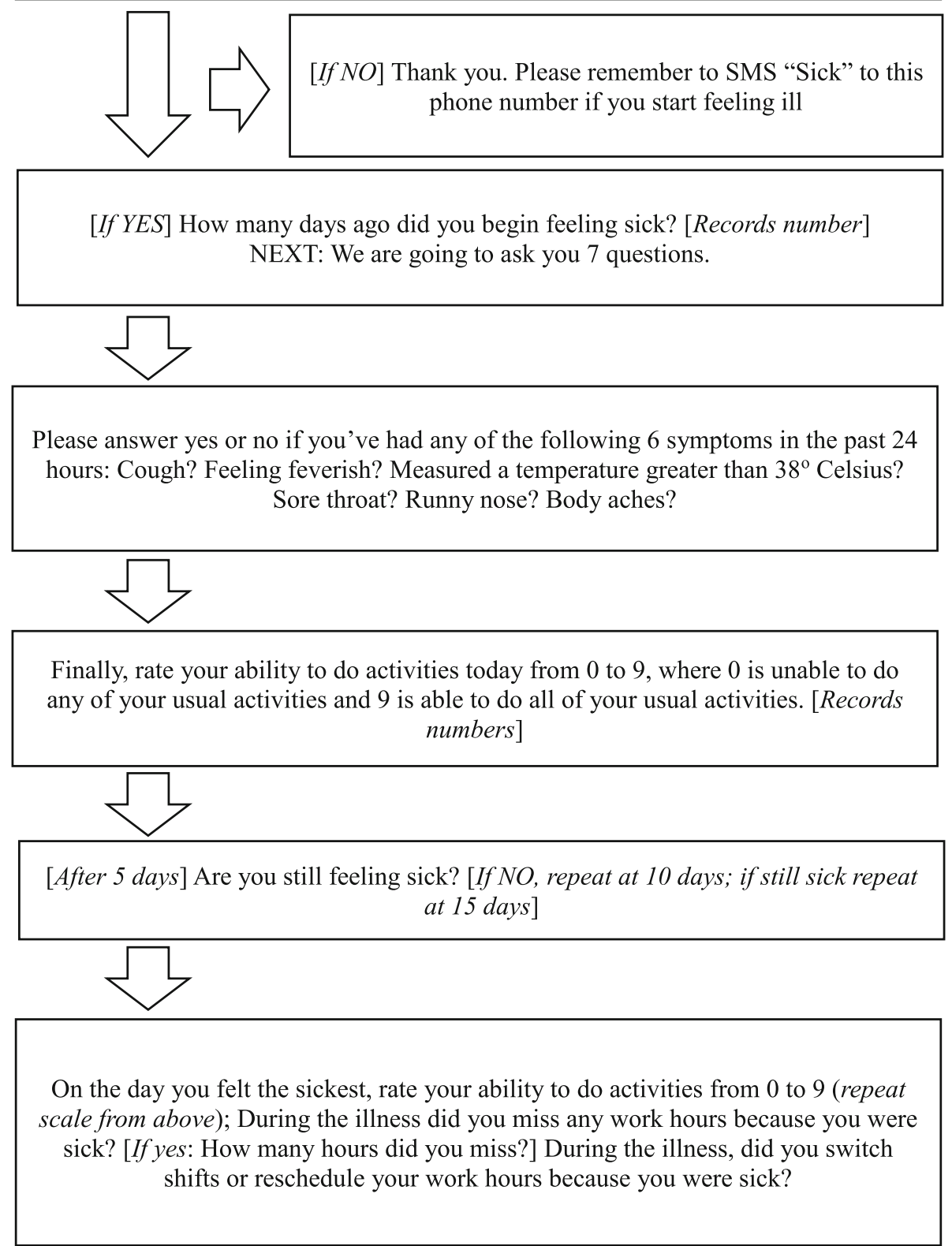

Fig. 2 Active Surveillance SMS Messaging Flow

swab, and a tube with room temperature transport medium. Respiratory specimens are placed in viral transport medium and delivered to the respective hospitals from HCP's residences by a courier service or are hand-carried by participants to study staff at their respective hospitals. If participants prefer not to collect the nasal swab themselves, study staff are able to collect it for them.

\section{Molecular diagnostics}

Specimens from Beilinson Hospital are stored at $4{ }^{\circ} \mathrm{C}$ and delivered by courier to the Clinical Virology Laboratory $(\mathrm{CVL})$ at Soroka University Medical Center twice a week, where they are immediately aliquoted and frozen at $-80^{\circ} \mathrm{C}$. Specimens from participating Soroka University Medical Center HCP are brought to the laboratory daily, where they are aliquoted and frozen. 
Table 3 Key Variables and Sources of Information for Participants

\begin{tabular}{|c|c|c|c|c|c|c|}
\hline & \multicolumn{2}{|l|}{ Self-reported } & \multicolumn{2}{|c|}{$\begin{array}{l}\text { Electronic Medical } \\
\text { Records }\end{array}$} & \multicolumn{2}{|l|}{ Time Period } \\
\hline & $\begin{array}{l}\text { Enrollment } \\
\text { survey }\end{array}$ & $\begin{array}{l}\text { EOS } \\
\text { survey }\end{array}$ & $\overline{E M R}$ & $\begin{array}{l}\text { Employee } \\
\text { Records }\end{array}$ & $\begin{array}{l}\text { From year(s) } \\
\text { prior to the study }\end{array}$ & $\begin{array}{l}\text { Data from years } \\
\text { enrolled in study }\end{array}$ \\
\hline \multicolumn{7}{|l|}{ Demographic } \\
\hline Sex & & & & & $\checkmark$ & \\
\hline Date of birth & & & & & $\checkmark$ & \\
\hline Marital status & $\checkmark$ & & $\checkmark$ & & & $\checkmark$ \\
\hline Country of birth & & & $\checkmark$ & & $\checkmark$ & \\
\hline Immigration date & & & $\checkmark$ & & $\checkmark$ & \\
\hline $\begin{array}{l}\text { Ethnicity by country of birth of individual, the parents, or } \\
\text { grandparents }\end{array}$ & & & $\checkmark$ & & $\checkmark$ & \\
\hline Socio-economic status by clinic address & & & $\checkmark$ & & $\checkmark$ & $\checkmark$ \\
\hline Supplementary insurance status & & & $\checkmark$ & & $\checkmark$ & \\
\hline Dates enrolled as CHS member & & & $\checkmark$ & & $\checkmark$ & \\
\hline Level of education & $\checkmark$ & & & & & $\checkmark$ \\
\hline $\begin{array}{l}\text { Household composition (number of rooms; } \\
\text { number of family members in the house) }\end{array}$ & $\checkmark$ & & & & & $\checkmark$ \\
\hline Occupation and work responsibilities & $\checkmark$ & & & & $\checkmark$ & $\checkmark$ \\
\hline Family income & $\checkmark$ & & & & & $\checkmark$ \\
\hline \multicolumn{7}{|l|}{ Health Status and Risk Behaviors } \\
\hline Health status and health behaviors & $\checkmark$ & $\checkmark$ & & & $\checkmark$ & \\
\hline Smoking status, history & & & $\checkmark$ & & $\checkmark$ & \\
\hline Pack years & & & $\checkmark$ & & $\checkmark$ & \\
\hline Height & & & $\checkmark$ & & $\checkmark$ & \\
\hline Weight & & & $\checkmark$ & & $\checkmark$ & \\
\hline Body mass index & & & $\checkmark$ & & $\checkmark$ & \\
\hline $\begin{array}{l}\text { Medication use for chronic conditions and } \\
\text { immunosuppressants }\end{array}$ & & & $\checkmark$ & & $\checkmark$ & \\
\hline \multicolumn{7}{|l|}{ Attitudes } \\
\hline Perceptions of illness, vaccines, missing work & $\checkmark$ & & & & & $\checkmark$ \\
\hline $\begin{array}{l}\text { Recollection of influenza vaccination } \\
\text { (for vaccinated } \mathrm{HCP} \text { ) }\end{array}$ & & $\checkmark$ & & & & $\checkmark$ \\
\hline $\begin{array}{l}\text { Reasons for not receiving the influenza } \\
\text { vaccine (for unvaccinated } \mathrm{HCP} \text { ) }\end{array}$ & & $\checkmark$ & & & & $\checkmark$ \\
\hline Job satisfaction & $\checkmark$ & & & & & $\checkmark$ \\
\hline \multicolumn{7}{|l|}{ Influenza Vaccination Documentation } \\
\hline Vaccine administration date & & & $\checkmark$ & & $\checkmark$ & $\checkmark$ \\
\hline Vaccine type & & & $\checkmark$ & & $\checkmark$ & $\checkmark$ \\
\hline Vaccine manufacturer \& lot & & & $\checkmark$ & & $\checkmark$ & $\checkmark$ \\
\hline Employee Records of Illness Absences & & & & $\checkmark$ & & $\checkmark$ \\
\hline \multicolumn{7}{|l|}{ Acute Respiratory IIIness } \\
\hline $\begin{array}{l}\text { Number of inpatient admissions associated } \\
\text { with acute illness }\end{array}$ & & & $\checkmark$ & & $\checkmark$ & $\checkmark$ \\
\hline \multicolumn{7}{|l|}{ Chronic Medical Conditions and Pregnancy } \\
\hline $\begin{array}{l}\text { Number of ambulatory or inpatient medical } \\
\text { encounters associated with chronic medical } \\
\text { condition }\end{array}$ & & & $\checkmark$ & & $\checkmark$ & $\checkmark$ \\
\hline Chronic medical conditions & & & $\checkmark$ & & $\checkmark$ & \\
\hline Pregnancy & & & & & $\checkmark$ & $\checkmark$ \\
\hline
\end{tabular}


After one freeze-thaw cycle, specimens are tested for influenza $\mathrm{A}$ viruses $[\mathrm{A}(\mathrm{H} 1 \mathrm{~N} 1) \mathrm{pdm} 09$ and $\mathrm{A}(\mathrm{H} 3 \mathrm{~N} 2)]$, influenza B viruses, respiratory syncytial virus (RSV), human metapneumovirus, and coronaviruses (NL63, 229E, OC43, HKU1) using validated multiplex quantitative real-time reverse transcription polymerase chain reaction (mqRT-PCR) assays [32], with protocols, primers, probes, and reagents supplied by Hy-labs (Israel) and Integrated DNA Technologies (USA). The CVL, in collaboration with the University of Michigan (Ann Arbor, Michigan, US), has completed the World Health Organization and US CDC influenza proficiency panels. Quality Control for Molecular Diagnostics (Glasgow, Scotland, UK) proficiency panels were also completed for influenza, RSV, human metapneumovirus (hMPV) and coronaviruses.

Some specimens will have molecular characterization with genetic sequencing and other assays to detect genetic markers to determine viral subclades and antiviral resistance at a reference laboratory approved by the study steering committee. Remaining aliquots of all study specimens may be sent to a US CDC-designated facility (also approved by the study steering committee) for additional virus characterization, banking, and storage. No specimens will contain personal identifiers.

Blood Specimens At enrollment, prior to each influenza season, $10 \mathrm{~mL}$ specimens of whole blood are collected from all participants. In addition, $5 \mathrm{~mL}$ specimens of whole blood are collected at the end of season 1 and the start and end of subsequent seasons. Participants who received the influenza vaccine during the study period will be asked to provide an additional sample of $5 \mathrm{~mL}$ of whole blood approximately 28 days (within a range of 21-42 days) after vaccination. Sera are extracted from whole blood and stored frozen until testing.

Participants who consent to providing additional blood at enrollment, as an optional part of the study, provide an additional $10 \mathrm{~mL}$ heparinized whole blood at enrollment and the end of season for extraction of peripheral blood mononuclear cells (PBMC). In addition, we collect $10 \mathrm{~mL}$ of heparinized whole blood approximately 7 days after vaccination from participants who agree to this optional part of the study. All PBMC samples are centrifuged, undergo cell count, diluted to $1-5 \times 10^{6} \mathrm{cells} / \mathrm{ml}$ and gradually frozen to $-80{ }^{\circ} \mathrm{C}$, and placed in liquid nitrogen within $24 \mathrm{~h}$. PBMC samples will be used for cell-mediated immunity assays.

\section{Hemagglutination inhibition assay}

The hemagglutination inhibition (HI) assay will be used to detect the presence of influenza antibodies in serum. $\mathrm{HI}$ to inactivated influenza vaccine components and influenza circulating strains will be performed at a reference laboratory using standard methods [33] as described previously [28, 34] (See Additional file 1: Annex 4). Egg-grown viruses will be supplied by the US CDC's International Reagent Resource. Preparation of serum samples will include (a) treatment with a receptor-destroying enzyme to remove nonspecific inhibitors, and (b) removal of nonspecific agglutinins by serum adsorption with packed red blood cells $(\mathrm{RBC})$. Standard $0.5 \%$ turkey $\mathrm{RBC}$ will be prepared for influenza A (H1N1)pdm09 antigens and ether-treated B influenza antigens. Given indications that neuraminidase of circulating antigenic clusters of influenza $\mathrm{A}(\mathrm{H} 3 \mathrm{~N} 2)$ viruses (since 2014) have acquired the ability to bind to $\mathrm{RBC}$, modified $\mathrm{HI}$ assays will be conducted for influenza $\mathrm{A}(\mathrm{H} 3 \mathrm{~N} 2)$ antigens using guinea pig red blood cells in the presence of the antiviral oseltamivir carboxylate, which inhibits influenza neuraminidase. Serum will be diluted 2-fold starting from 1:10. The HI titer will be the reciprocal of the serum dilution in the last well with complete HI. The geometric mean titer from duplicate results will be reported; $\mathrm{HI}<10$ will be considered 5 for the purposes of statistical analyses.

\section{Additional immunological assays}

Additional serologic testing may occur, including neuraminidase inhibition assay testing, antigen microarray testing [35] and other approaches. Neuraminidase-specific antibodies have been shown to play a role in protection against influenza infection [36, 37]. However, many questions remain about the role of neuraminidase in vaccine effectiveness [38].

\section{Attitudes toward morbidity due to influenza and other respiratory illnesses}

As a sub-study within this project, approximately 15-25 in-depth open-ended qualitative interviews are conducted each year with participants who recently had a wintertime respiratory illness and agree to participate in this sub-study.

Participants are contacted by phone approximately 1 week after confirmation of respiratory illness resolution and asked to participate in an interview. Interviews cover topics including illness experience, including symptoms, duration, perceived severity, and disruption to their daily activities and responsibilities, perceptions of how the illness impacted work responsibilities, perceptions of how the illness impacted life outside of work, and reasons for choosing to receive or not receive the influenza vaccine this season (see Additional file 1: Annex 5). Interviews will be recorded by audio recorder and later transcribed. After transcription, the data are translated from Hebrew to English and qualitatively analyzed using NVivo Software (QSR International, Melbourne, Australia) to identify themed code-words 
that may point to common patterns of comments across the interviews.

\section{Data management REDCap}

Data collection and site-level management are conducted using REDCap, a browser-based metadata-driven software system (Vanderbilt University, Nashville, TN, USA) [39]. Most study instruments, including the recruitment log, online surveys, and laboratory results, allow for real-time data entry directly into REDCap. Surveys are designed to be self-administered electronically through the Internet using home computers, personal mobile telephones, or on computers or tablets provided at the workplace by study staff. In addition, study staff enter participants' responses directly into the REDCap database for interviews administered by telephone. Routine quality assurance monitoring is conducted locally by the project manager and centrally by the data coordinator (Abt Associates). Missing or unclear information is corrected through follow-up contact with participants.

\section{Textlt}

TextIt (TextIt, Kigali, Rwanda) is an online platform that uses logic flows to send tailored SMS messages to participants. Custom decision trees were developed in order to trigger exchanges with participants during active surveillance (described above). Specifically, TextIt documents answers to weekly illness inquiries, dates of illness onset and resolution, ratings of symptoms, and missed work. Study staff perform bi-weekly exports of TextIt data and import the data into an Excel macro-enabled workbook in order to track participant responses and illness events over time (see Additional file 1: Annex 6 for more details about TextIt).

\section{Statistical considerations Cohort size}

The required number of participants depends on multiple factors, including expected influenza attack rate, vaccination coverage, and anticipated study attrition. We estimated that with 2340 participants contributing 5 months at-risk for influenza infection per season, $40 \%$ vaccine coverage, and $10 \%$ influenza illness attack rate, we would be powered to estimate a true vaccine effectiveness of $50 \%$ with confidence intervals that do not overlap with zero.

\section{Sample size for incidence calculations}

The full cohort provides the sample needs for estimating the frequency (or incidence within the study sample) of influenza illness. Given the larger sample demands for the vaccine effectiveness objectives, we expect to have ample statistical power for most objectives that involve estimating incidence or frequencies.

\section{Sample size for immune response to vaccine and infection objectives}

To assess the immune response to the vaccine, we plan to collect serum from the cohort members prior to vaccine availability, after vaccination, and at the end of each season. Our study design mirrors approaches used in previous studies that assessed the $\mathrm{HI}$ antibody titers among HCP analyzed sera from subgroups of 300-800 participants who provided sera at the same three time points [28]. See Additional file 1: Annex 7 for more details.

\section{Data analysis of VE}

Rates of acute illness associated with mqRT-PCR confirmed influenza virus infection (influenza illness) will be calculated as the number of influenza illnesses divided by person-time measured in weeks of active surveillance. Regression models will be used to estimate influenza vaccine effectiveness ( 1 - rate of influenza illness among vaccinated $\mathrm{HCP} /$ rate among unvaccinated $\mathrm{HCP} * 100)$ with $95 \%$ confidence intervals. Adjusted models of influenza vaccine effectiveness will include study year, calendar time (i.e., weeks between illness onset and week of peak of influenza season), and a propensity for vaccination score calculated using multivariable logistic regression [40]. Other potential confounders, including study site, will be examined and included in the adjusted model if they change IVE point estimates by $>5 \%$.

\section{Data analysis of immunogenicity}

Since distributions of HI titer data are typically highly left-skewed, all statistical analyses will be conducted using log base- 2 transformed titer data; results are then back-transformed to the original scale for ease of interpretation $[28,41]$. Pre- and post-vaccine draws are assumed to be correlated within each person, thus repeated measures linear mixed models will be fitted to estimate geometric mean titers (GMT) and geometric mean ratios (GMR). Compound symmetric covariance error structures will be assumed for repeated measures within individuals. GMT will be calculated by back-transforming the least squares mean estimates of logged titer data. GMR will be calculated by back-transforming the difference of least squares means of post-vaccination and pre-vaccination logged titer estimates. GMR will be interpreted as the geometric mean fold ratio of post-vaccination titer to pre-vaccination titer. Multivariate estimates adjusted a priori for age and sex. Linear, quadractic, and cubic terms for age will be examined to consider possible nonlinear associations with age. Other covariates (e.g., education, household size, working in a hospital setting) may also be adjusted within multivariable models if they were associated with the number of 
prior vaccinations and either preseason GMT or post-vaccination GMR among vaccines.

\section{Ethical considerations}

The study protocol and procedures have been reviewed and approved by the Helsinki committees (institutional review board) at both of the study sites, and by Abt Associates (the coordinating institution on which US CDC relies). In addition, extraction of data from the CHS EMR was approved by the Data Use Committee of the Clalit Research Institute.

All participants complete written informed consent in Hebrew. Small gifts (such as a gift card) are given to participants at study milestones like completion of enrollment, blood draws, and completion of end-of-season survey. Influenza test results are given to those participants who ask to receive them. Given the research nature of the laboratory methods and time delays in batch testing, mqRT-PCR findings are not available to inform clinical decisions.

\section{Discussion}

We described the recruitment, enrollment, active surveillance, data collection, laboratory methods, and data management procedures for the SHIRI cohort - a US CDC-sponsored prospective study, overseen by CHS, of approximately $2300 \mathrm{HCP}$ at two hospitals in Israel. In this study, we will describe the frequency of influenza virus infections among HCP in Israel. Using a broad case definition of respiratory illness, we aim to identify a wide range of ARFI in order to assess more accurately the burden of respiratory illness among HCP. Due to CHS's extensive EMR, we will have the ability to look at HCP influenza vaccination records up to 10 years prior to study enrollment in order to better understand how HI antibody titers differ depending on the number and specific combinations of prior influenza vaccinations. We will also be able to evaluate influenza vaccine effectiveness in preventing medically attended and non-medically attended influenza illness and associated missed work days.

The findings from the SHIRI cohort will increase our understanding of the burden of acute respiratory illnesses, and influenza virus illness specifically, among HCP in Israel. The unique design of the study, which includes comprehensive medical and vaccination data, active surveillance using a broad case definition, and both molecular and serologic diagnostics, will provide important data on influenza vaccine immunogenicity and effectiveness among HCP. The combination of insights about influenza burden and vaccine effectiveness may potentially inform influenza vaccine policy for HCP in Israel and internationally.

\section{Strengths}

Our study benefits from the fact that all enrolled HCP are members of $\mathrm{CHS}$, and therefore extensive information is recorded in the EMR, which provides real-time data about ambulatory visits, hospitalizations, and medication use. In addition, the EMR provides reliable historic data about the participants, such as prior vaccination information, socio-economic variables, and data about health status and health behaviors. In our study, even if an ARFI is not reported through the SMS surveillance system, daily monitoring of the EMR for medical visits and medications will allow us to identify illnesses and contact participants directly in order to collect respiratory specimens and complete the illness survey. Combining our study with EMR data and data from the two hospitals' human resources departments will also allow us to reliably map the impact of illness on missed work and to evaluate days worked when ill (presenteeism).

In addition, the broad case definition of an ARFI promises to be more sensitive for influenza illness than case definitions used in previous HCP research [24] and should allow us to characterize a continuum of mild to moderately severe illnesses. Another strength is that the two study sites (Soroka University Medical Center and Beilinson Hospital) are among the largest hospitals in Israel, and the populations they serve and the HCP they employ vary in socio-demographic and economic characteristics. Soroka University Medical Center, the only general hospital in the Negev region in southern Israel, serves two main local populations: Jews, who mostly live in urban settings, and Bedouin Arabs, who live in a range of settlement types, from cities to unrecognized rural villages that lack electricity and running water [42, 43]. Beilinson Hospital, located in central Israel, serves a mostly urban population of a relatively higher socio-economic status compared to Soroka University Medical Center. Conducting our study in these two different settings may allow us to identify socio-demographic differences in KAP, vaccine uptake, and influenza illness attack rates. The use of mixed methods, including laboratory, clinical and epidemiological quantitative data, and in-depth qualitative interviews, creates a comprehensive approach, which is particularly important when trying to understand issues of influenza vaccine compliance and hesitancy. Finally, the study cohort includes several unique sub-studies.

\section{Limitations}

Our study has at least four limitations. First, our ability to generalize vaccine effectiveness findings from the study years to the potential preventive value of an influenza vaccine program may be limited. The effectiveness of the vaccine depends in part on the types of viruses circulating and the antigenic and genetic match between 
vaccine components and circulating strains in a given year. The precision of the estimates will also depend on the number of influenza cases.

Second, we will be cautious in interpreting self-reported information, given potential biases in recall and self-presentation. While some of the information collected through active surveillance can be verified with administrative data, other information, such as symptoms for non-medically attended illnesses, cannot be verified by another data source.

Third, although the random stratified sampling design intentionally includes HCP with a mixture of characteristics and work responsibilities, there are relatively few HCP in some of the strata. For example, few male medical assistants and support personnel are employed at the two hospitals, and therefore it is unlikely that we will be able to enroll large numbers from this category. This may limit our ability to examine the association between the frequency of influenza illness and specific combinations of age, sex, and occupation.

Fourth, during the third wave of recruitment we accept volunteers, regardless of age, profession, or vaccine status, which can present a sampling bias. However, characteristics of these volunteer participants will be compared with those of participants recruited during the first two waves in order to evaluate potential differences between the groups.

\section{Additional file}

Additional file 1: Description of additional terms and methods used in the Study of Healthcare Personnel with Influenza and other Respiratory Viruses in Israel. (DOCX $77 \mathrm{~kb}$ )

\begin{abstract}
Abbreviations
ARFI: Acute respiratory illness or febrile illness; CHS: Clalit Health Services; CVL: Clinical Virology Laboratory; EMR: Electronic medical record; GMT: Geometric mean titer; GMR: Geometric mean ratios; HCP: Healthcare personnel; HI: Hemagglutination inhibition; hMPV: Human metapneumovirus; ICD-9: The international classification of diseases, ninth revision; ICPC: International Classification of Primary Care Coding; IVE: Influenza vaccine effectiveness; KAP: Knowledge, attitudes, and practices; mqRTPCR: Multiplex quantitative real-time reverse transcription polymerase chain reaction; PBMC: Peripheral blood mononuclear cells; RBC: Red blood cells; REDCap: Research Electronic Data Capture; RSV: Respiratory syncytial virus; SHIRI: Study of Healthcare Personnel with Influenza and other Respiratory Viruses in Israel; SMS: Short message service; US CDC: United States Centers for Disease Control and Prevention
\end{abstract}

\section{Acknowledgments}

The authors would like to thank Becca Feldman (Clalit Research Institute) for epidemiological support, Nataliya Sokolski (Clalit Research Institute) for administrative support, Sydney Krispin (Clalit Research Institute) for assistance in editing and reviewing the manuscript, Orly Barashi Zamir (Soroka Universitty Medical Center) for on-site study coordination, and Jacob Dreiher (Soroka University Medical Center) for on-site study leadership. The authors would like to thank Sonja Olsen, Jerome Tokars, and Meredith McMorrow for feedback on an earlier version of this manuscript (US CDC). We would like to thank the study staff at all participating hospitals. Finally, we are extremely grateful to the study participants.

\section{Funding}

Contract HHSD2002013M53890B within CDC's Achieving Public Health Impact through Research and task 200-2014-F-60406 ("The Epidemiology and Prevention of Influenza Virus Infections in Low- and Middle-Income Countries") to Abt Associates from US CDC.

\section{Availability of data and materials}

Not applicable.

\section{Authors' contributions}

Study concept and design: MGT, RB, ASM, REM, ALP, DG, RW, GNA, MLR, and ND; Laboratory methods: YAS, ETM, MGT; Literature search: MGT, MAK, AH; Acquisition of data: IG, WC; Coordination of the study: AH, MAK; Wrote the manuscript: $\mathrm{AH}, \mathrm{MAK}, \mathrm{MGT}, \mathrm{RW}$; Drafting of the manuscript and critical revision of the manuscript for important intellectual content: $A L P, D G, R W$, YSA, GNA, IG, MLR, ND, AR, RGA, TH, AGF, ASM, EAB, JMF, ETM REM, JMNQ, $M L, W C, R B ; A l l$ authors read and approved the final manuscript.

\section{Ethics approval and consent to participate}

The study protocol and procedures were reviewed and approved by Institutional Review Boards at Abt Associates (the coordinating institution on which US CDC relies) and at each study site: Soroka University Medical Center and Beilinson Hospital. All participants complete written informed consent.

\section{Consent for publication}

Not applicable.

\section{Competing interests}

No conflicts of interest were reported. The findings and conclusions in this report are those of the authors and do not necessarily represent the official position of the US CDC.

\section{Publisher's Note}

Springer Nature remains neutral with regard to jurisdictional claims in published maps and institutional affiliations.

\section{Author details}

${ }^{1}$ Chief Physician's Office, Clalit Health Services, Clalit Research Institute, Tel Aviv, Israel. ${ }^{2}$ School of Public Health, Medical School for International Health, Faculty of Health Sciences, Ben Gurion University of the Negev, Beer Sheva, Israel. ${ }^{3}$ Department of Epidemiology, University of Michigan School of Public Health, Ann Arbor, MI, USA. ${ }^{4}$ Rabin Medical Center, Occupational Medicine Department, Petah Tikva, Israel. ${ }^{5}$ Pediatric Infectious Disease Unit, Soroka University Medical Center, Beer Sheva, Israel. ${ }^{6}$ Abt Associates, Inc, Atlanta, GA, USA. ${ }^{7}$ Clinical Virology, Soroka University Medical Center, Ben Gurion University of the Negev, Beer Sheva, Israel. ${ }^{8}$ Department of Health Systems Management, School of Public Health, Faculty of Health Sciences, Ben Gurion University of the Negev, Beer Sheva, Israel. ${ }^{9}$ Department of Microbiology Immunology and Genetics, Faculty of Health Sciences, Ben Gurion University of the Negev, Beer Sheva, Israel. ${ }^{10}$ Vaccine and Infectious Disease Division, Fred Hutch Cancer Research Center, Seattle, WA, USA. ${ }^{11}$ Israel Center for Disease Control, Ministry of Health, Tel Hashomer, Ramat Gan, Israel.

${ }^{12}$ Influenza Division, Centers for Disease Control and Prevention (CDC), Atlanta, GA, USA. ${ }^{13}$ U.S. Naval Medical Research Unit N 6 - Lima, Lima, Peru.

${ }^{14}$ Department of Epidemiology and Preventive Medicine, School of Public Health, Sackler Faculty of Medicine, Tel Aviv University, Tel Aviv, Israel.

Received: 7 March 2018 Accepted: 16 October 2018

Published online: 06 November 2018

\section{References}

1. Kuster SP, Shah PS, Coleman BL, Lam PP, Tong A, Wormsbecker A, et al. Incidence of influenza in healthy adults and healthcare workers: a systematic review and meta-analysis. PLoS One. 2011;6. https://doi.org/10. 1371/journal.pone.0026239.

2. Brankston G, Gitterman L, Hirji Z, Lemieux C, Gardam M. Transmission of influenza a in human beings. Lancet Infect Dis. 2007;7:257-65. https://doi. org/10.1016/S1473-3099(07)70029-4.

3. Thomas RE. Do we have enough evidence how seasonal influenza is transmitted and can be prevented in hospitals to implement a 
comprehensive policy? Vaccine. 2016;34:3014-21. https://doi.org/10.1016/j. vaccine.2016.04.096.

4. Tran K, Cimon K, Severn M, Pessoa-Silva CL, Conly J. Aerosol generating procedures and risk of transmission of acute respiratory infections to healthcare workers: a systematic review. PLoS One. 2012;7. https://doi.org/ 10.1371/journal.pone.0035797.

5. Molinari NAM, Ortega-Sanchez IR, Messonnier ML, Thompson WW, Wortley PM, Weintraub E, et al. The annual impact of seasonal influenza in the US: measuring disease burden and costs. Vaccine. 2007;25:5086-96. https://doi. org/10.1016/j.vaccine.2007.03.046.

6. Pearson ML, Bridges $\mathrm{CB}$, Harper $\mathrm{S}$ a. Influenza vaccination of health-care personnel: recommendations of the healthcare infection control practices advisory committee (HICPAC) and the advisory committee on immunization practices (ACIP). MMWR Recomm Rep. 2006:55:1-16.

7. Vanhems P, Voirin N, Roche S, Escuret V, Regis C, Gorain C, et al. Risk of influenza-like illness in an acute health care setting during community influenza epidemics in 2004-2005, 2005-2006, and 2006-2007: a prospective study. Arch Intern Med. 2011;171:151-7. https://doi.org/10.1001/archinternmed. 2010.500.

8. De Serres G, Skowronski DM, Ward BJ, Gardam M, Lemieux C, Yassi A, et al. Influenza vaccination of healthcare workers: critical analysis of the evidence for patient benefit underpinning policies of enforcement. PLoS One. 2017; 12. https://doi.org/10.1371/journal.pone.0163586.

9. Carrat F, Vergu E, Ferguson NM, Lemaitre M, Cauchemez S, Leach S, et al. Time lines of infection and disease in human influenza: a review of volunteer challenge studies. Am J Epidemiol. 2008;167:775-85. https://doi. org/10.1093/aje/kwm375.

10. Poland GA, Tosh P, Jacobson RM. Requiring influenza vaccination for health care workers: seven truths we must accept. Vaccine. 2005;23:2251-5. https://doi.org/10.1016/j.vaccine.2005.01.043.

11. Rakita RM, Hagar B a, Crome P, Lammert JK. Mandatory influenza vaccination of healthcare workers: a 5-year study. Infect Control Hosp Epidemiol. 2010;31:881-8. https://doi.org/10.1086/656210.

12. Abu-Gharbieh E, Fahmy S, Rasool BA, Khan S. Influenza vaccination: healthcare workers attitude in three Middle East countries. Int J Med Sci. 2010;7:319-25.

13. Hollmeyer H, Hayden F, Mounts A, Buchholz U. Review: interventions to increase influenza vaccination among healthcare workers in hospitals. Influenza Other Respir Viruses. 2013;7:604-21. https://doi.org/10.1111/irv.12002.

14. Hollmeyer HG, Hayden F, Poland G, Buchholz U. Influenza vaccination of health care workers in hospitals-a review of studies on attitudes and predictors. Vaccine. 2009;27:3935-44. https://doi.org/10.1016/j.vaccine.2009. 03.056.

15. Naleway AL, Henkle EM, Ball S, Bozeman S, Gaglani MJ, Kennedy ED, et al. Barriers and facilitators to influenza vaccination and vaccine coverage in a cohort of health care personnel. Am J Infect Control. 2014;42:371-5. https://doi.org/10.1016/j.ajic.2013.11.003.

16. Thompson MG, Gaglani MJ, Naleway A, Ball S, Henkle EM, Sokolow LZ, et al. The expected emotional benefits of influenza vaccination strongly affect pre-season intentions and subsequent vaccination among healthcare personnel. Vaccine. 2012;30:3557-65. https://doi.org/10.1016/.vaccine.2012.03.062.

17. Influenza vaccination of Medical Teams n.d. https://www.health.gov.il/ PublicationsFiles/Flu_stuff2015_2016.pdf.

18. Osterholm MT, Kelley NS, Sommer A, Belongia EA. Efficacy and effectiveness of influenza vaccines: a systematic review and meta-analysis. Lancet Infect Dis. 2012;12:36-44. https://doi.org/10.1016/S1473-3099(11)70295-X.

19. Wilde JA, McMillan JA, Serwint J, Butta J, O'Riordan MA, Steinhoff MC. Effectiveness of influenza vaccine in health care professionals: a randomized trial. JAMA. 1999;281:908-13. https://doi.org/10.1001/jama.281.10.908.

20. Petrie JG, Ohmit SE, Johnson E, Cross RT, Monto AS. Efficacy studies of influenza vaccines: effect of end points used and characteristics of vaccine failures. J Infect Dis. 2011;203:1309-15. https://doi.org/10.1093/infdis/jir015.

21. Thompson MG, Gaglani MJ, Naleway AL, Dowell SH, Spencer S, Ball S, et al. Reduced serologic sensitivity to influenza a virus illness among inactivated influenza vaccinees. Vaccine. 2016;34:3443-6. https://doi.org/10.1016/j. vaccine.2016.04.085.

22. Thomas RE, Jefferson T, Lasserson TJ. Influenza vaccination for healthcare workers who work with the elderly: systematic review. Vaccine. 2010;29:344-56. https://doi.org/10.1016/j.vaccine.2010.09.085.

23. Thomas RE, Jefferson $T$, Lasserson TJ. Influenza vaccination for healthcare workers who care for people aged 60 or older living in long-term care institutions. Cochrane Database Syst Rev. 2013;7:CD005187. https://doi.org/ 10.1002/14651858.CD005187.pub4.

24. Henkle E, Irving S a, Naleway AL, Gaglani MJ, Ball S, Spencer S, et al. Comparison of laboratory-confirmed influenza and noninfluenza acute respiratory illness in healthcare personnel during the 2010-2011 influenza season. Infect Control Hosp Epidemiol. 2014;35:538-46. https://doi.org/10. 1086/675832.

25. Ng a NM, Lai CKY. Effectiveness of seasonal influenza vaccination in healthcare workers: a systematic review. J Hosp Infect. 2011;79:279-86. https://doi.org/10.1016/j.jhin.2011.08.004.

26. Kenah E, Chao DL, Matrajt L, Halloran ME, Longini IM. The global transmission and control of influenza. PLoS One. 2011;6. https://doi.org/10. 1371/journal.pone.0019515.

27. McLean HQ, Thompson MG, Sundaram ME, Meece JK, McClure DL, Friedrich $T C$, et al. Impact of repeated vaccination on vaccine effectiveness against influenza A(H3N2) and B during 8 seasons. Clin Infect Dis. 2014;59:1375-85. https://doi.org/10.1093/cid/ciu680.

28. Gaglani M, Spencer S, Ball S, Song J, Naleway A, Henkle E, et al. Antibody response to influenza $A(\mathrm{H} 1 \mathrm{~N} 1)$ pdm09 among healthcare personnel receiving trivalent inactivated vaccine: effect of prior monovalent inactivated vaccine. J Infect Dis. 2014;209:1705-14. https://doi.org/10.1093/ infdis/jit825.

29. Belongia EA, Skowronski DM, McLean HQ, Chambers C, Sundaram ME, De Serres G. Repeated annual influenza vaccination and vaccine effectiveness: Review of evidence. Expert Rev Vaccines 2017:14760584.2017.1334554. doi: https://doi.org/10.1080/14760584.2017.1334554.

30. Melia M, O'Neill S, Calderon S, Hewitt S, Orlando K, Bithell-Taylor K, et al. Development of a flexible, computerized database to prioritize, record, and report influenza vaccination rates for healthcare personnel. Infect Control Hosp Epidemiol. 2009;30:361-9. https://doi.org/10.1086/596043.

31. Thompson MG, Ferber JR, Odouli R, David D, Shifflett P, Meece JK, et al. Results of a pilot study using self-collected mid-turbinate nasal swabs for detection of influenza virus infection among pregnant women. Influenza Other Respir Viruses. 2015;9:155-60. https://doi.org/10.1111/irv.12309.

32. Lieberman D, Lieberman D, Shimoni A, Keren-Naus A, Steinberg R, ShemerAvni Y. Identification of respiratory viruses in adults: nasopharyngeal versus oropharyngeal sampling. J Clin Microbiol. 2009;47:3439-43. https://doi.org/ 10.1128/JCM.00886-09.

33. Who. Manual for the laboratory diagnosis and virological surveillance of influenza. World Heal Organ. 2011;2011:153.

34. Thompson MG, Naleway A, Fry AM, Ball S, Spencer SM, Reynolds S, et al. Effects of repeated annual inactivated influenza vaccination among healthcare personnel on serum hemagglutinin inhibition antibody response to a/Perth/16/2009 (H3N2)-like virus during 2010-11. Vaccine. 2016;34:981-8. https://doi.org/10.1016/j.vaccine.2015.10.119.

35. Hertz T, Shagal A, Ohmit S. Immune history to influenza infection and vaccination predicts antibody responses to the seasonal influenza vaccine. J Immunol. 2016;196(1 Supplement):146.24.

36. Couch RB, Atmar RL, Franco LM, Quarles JM, Wells J, Arden N, et al. Antibody correlates and predictors of immunity to naturally occurring influenza in humans and the importance of antibody to the neuraminidase. J Infect Dis. 2013;207:974-81. https://doi.org/10.1093/infdis/jis935.

37. Monto AS, Petrie JG, Cross RT, Johnson E, Liu M, Zhong W, et al. Antibody to influenza virus neuraminidase: an independent correlate of protection. J Infect Dis. 2015;212:1191-9. https://doi.org/10.1093/infdis/jiv195.

38. Laguio-Vila MR, Thompson MG, Reynolds S, Spencer SM, Gaglani M, Naleway A, et al. Comparison of serum hemagglutinin and neuraminidase inhibition antibodies after 2010-2011 trivalent inactivated influenza vaccination in healthcare personnel. Open Forum Infect Dis. 2015;2 https://doi.org/10.1093/ofid/ofu1 15.

39. Harris PA, Taylor R, Thielke R, Payne J, Gonzalez N, Conde JG. Research electronic data capture (REDCap)-A metadata-driven methodology and workflow process for providing translational research informatics support. J Biomed Inform. 2009;42:377-81. https://doi.org/10.1016/j.jbi.2008.08.010.

40. D'Agostino RB Jr, D'Agostino RBS. Estimating treatment effects using observational data. JAMA. 2007;297:314-6.

41. Coudeville L, Bailleux F, Riche B, Megas F, Andre P, Ecochard R. Relationship between haemagglutination-inhibiting antibody titres and clinical protection against influenza: development and application of a bayesian random-effects model. BMC Med Res Methodol. 2010;10. https://doi.org/10. 1186/1471-2288-10-18 
42. Greenberg D, Givon-Lavi N, Newman N, Bar-Ziv J, Dagan R. Nasopharyngeal carriage of individual Streptococcus pneumoniae serotypes during pediatric pneumonia as a means to estimate serotype disease potential. Pediatr Infect Dis J. 2011;30:227-33. https://doi.org/10.1097/INF.0b013e3181f87802.

43. Abuhazira YS. The Bedouin population in IsraelPopulation register compared with population estimation as basis of demographic indexes. Jerusalem: CBS; 2010. http://cbs.gov.il/www/publications/pw50.pdf.

Ready to submit your research? Choose BMC and benefit from:

- fast, convenient online submission

- thorough peer review by experienced researchers in your field

- rapid publication on acceptance

- support for research data, including large and complex data types

- gold Open Access which fosters wider collaboration and increased citations

- maximum visibility for your research: over $100 \mathrm{M}$ website views per year

At $\mathrm{BMC}$, research is always in progress.

Learn more biomedcentral.com/submissions 